\section{Past imperfect}

Publications more than 25 years old are likely to be forgotten, which is a shameful waste.

THE letter from Professor Edward Harrison on page 574 of this issue should not be mistaken for yet another claim to priority for discovery (although it is that). The letter is more important for what it implies about the way in which even the relatively recent literature can quickly fail to serve as the vivid record of past discovery that it is generally supposed to be. The point at issue is the resolution of Olbers' paradox - the remark early in the nineteenth century that, if the number of stars in the Universe were so large as to be an approximation to infinity, the whole surface of even the night sky should be as bright as the surface of the Sun (or whatever is the brightness of a typical star).

Early in the nineteenth century, it had become clear that the number of observable stars increased with each improvement of technique, suggesting that the number might not be very different from infinity, but the notion that the Universe might be expanding, and that light from distant stars might on that account be shifted towards the less bright red end of the spectrum, had not yet arisen. So Olbers' paradox could be used in two ways - either to assert that the number of stars in the Universe (other galaxies had not then been discovered) could not, after all, be infinite or anything like it, or to focus attention on the need to explain why the night sky is not uniformly bright.

The doctrine of the expanding Universe radically changed the terms of reference in two ways. First, the brightness of receding stars will be diminished by the recession; second, an expanding universe must expand from something and so must have a finite age, raising the question whether there has been enough time since the beginning of the Universe for there to have been created enough stars (or galaxies) for the premise of Olbers' paradox (that the number of stars or galaxies is essentially infinite) to be satisfied. So the resolution of the paradox hinges on the relative importance of recession and finite age.

What Harrison now says is that, more than a quarter of a century ago, he had argued in a contribution published in this journal that the effect of recession is much less important than that of the finite age of the Universe. That is also the conclusion of an argument published earlier this year by Paul S. Wesson, which attracted an approving comment in Nature; neither Wesson's article nor the account of it in this journal referred to Harrison's much earlier work.

The tone of Harrison's moderate letter prompts one interesting conclusion - that even he is not surprised that a piece of work published in 1964 should have been forgotten by 1991. (It is more shocking that this journal's commentator should have failed to draw attention to
Harrison than that Wesson should not have done so.) But sadly, that must these days be a common experience. There is a sense in which the literature is rendered inaccessible by the mere passage of time. Of course, outstanding discoveries make their way into the textbooks and monographs from which young people learn science, but the likelihood that other material will be retrievable necessarily depends on the idiosyncracies of individual memories.

There is no early prospect that matters will improve. Although machine retrieval could in principle present interested readers with references to everything previously published in some nominated field, it is unthinkable that such a service would be feasible for the years before the creation of the first machine databases (in the early 1970s). And even then there are obvious intellectual difficulties, notably those of constructing cumulative indices that will retain their meaning as the decades pass. So it may be necessary that we should become reconciled to the inadvertent neglect of earlier work, despite the time and talent wasted in its duplication.

\section{Circles within circles}

Are the mysterious circles of flattened corn in British cornfields simply a media ploy?

IF ultimately of no other scientific interest, the annual spate of crop circles in British fields brings to light the circular nature of science itself - at least as far as the media are concerned. Stories have appeared over the summer in the British press declaring the file on the inscrutable circles closed at the hands of some expert or other. Strangely enough, however, the same theories had all been advanced at this time last year.

Most entertaining, and hence most appealing to a desperate editor, is the theory that the rings of flattened grain are carved by birds chasing each other round in circles as part of some seasonal mating ritual. No matter that the circles are all far too perfect, and that the process has never been observed. Animals are funny; sex is funny; run the story each year as a 'discovery'.

The same entertainment value obviously does not adhere to the proposition that the circles are some kind of electrostatic phenomenon brought about by an unknown, yet firmly prosaic, combination of climatic and geographical factors. It was a great relief to everybody, then, when it emerged that this year the boring explanation was to be investigated by a team of scientists armed with expensive photographic equipment and characteristically taking something too seriously. No surprise then that the electrostatic explanation cropped up again this summer.

Whatever the eventual outcome of the crop circle saga, it is unlikely to stand out from the perennial volley of recycled 'discoveries' in the British press, which shows no signs of abating in the years to come. 